\title{
EGFR as paradoxical predictor of chemosensitivity and outcome among triple-negative breast cancer
}

\author{
HIROKO NOGI $^{1}$, TADASHI KOBAYASHI ${ }^{2}$, MASAFUMI SUZUKI $^{3}$, ISAO TABEI $^{1}$, \\ KAZUMI KAWASE ${ }^{1}$, YASUO TORIUMI ${ }^{1}$, HISAKI FUKUSHIMA ${ }^{1}$ and KEN UCHIDA ${ }^{1}$
}

\begin{abstract}
Departments of ${ }^{1}$ Breast and Endocrine Surgery, ${ }^{2}$ Medical Oncology and Hematology and ${ }^{3}$ Pathology, The Jikei University School of Medicine, 3-25-8 Nishi-shinbashi, Minato-ku, Tokyo 105-8461, Japan
\end{abstract}

Received September 9, 2008; Accepted November 3, 2008

DOI: 10.3892/or_00000238

\begin{abstract}
We retrospectively analyzed the expression of epidermal growth factor receptor (EGFR) as a prognostic marker to predict neoadjuvant chemotherapy response and survival among breast cancer subtypes. We used immunohistochemical profiles to subtype the patients. EGFR expression was determined using immunohistochemistry. All patients received an anthracycline-based regimen preoperatively. Ninety-three patients also received docetaxel. Of the 117 patients tested, 28 (24\%) were triple-negative breast cancer (TNBC) and $73(62 \%)$ were hormone receptorpositive (luminal) subtype. Among the TNBC patients, a significantly higher incidence of EGFR expression (50\%) was observed $(\mathrm{P}=0.002)$, and EGFR expression was related to a less favorable response to chemotherapy $(\mathrm{P}=0.03)$ and poorer survival $(\mathrm{P}=0.17)$; in contrast, among the luminal subtype patients, positive EGFR expression was related to a favorable clinical response $(\mathrm{P}=0.06)$ and better survival $(\mathrm{P}=0.11)$. This retrospective analysis demonstrated that EGFR expression may represent an adverse prognostic marker in patients with TNBC and may provide a valuable tool for selecting appropriate treatment regimens for patients with TNBC.
\end{abstract}

\section{Introduction}

Triple-negative breast cancers (TNBC) are defined by a lack of estrogen receptor (ER), progesterone receptor (PgR) and human epidermal growth factor receptor 2 (HER2) expression. Therefore, TNBC is unlikely to benefit from anti-estrogen hormonal therapies or trastuzumab (1).

TNBC is reported to account for $10-15 \%$ of all sporadic breast cancers (2-5) and 23-32\% of relatively advanced breast cancers treated with neoadjuvant chemotherapy $(6,7)$.

Correspondence to: Dr Hiroko Nogi, Department of Breast and Endocrine Surgery, The Jikei University School of Medicine, 3-25-8 Nishi-shinbashi, Minato-ku, Tokyo 105-8461, Japan

E-mail: nogi_h@jikei.ac.jp

Key words: triple-negative breast cancer, epidermal growth factor receptor, neoadjuvant chemotherapy, luminal
Histologically, they are poorly differentiated tumors, and most fall into a basal-like subtype that is characterized by staining for basal markers (i.e., cytokeratin 5/6, 14 and 17) (1).

Three major subtypes (basal-like, HER2 overexpressing, and luminal) had been identified using gene expression analyses, and these subtypes have different prognoses $(8,9)$. Poorer outcomes are seen for the two hormone receptornegative types, compared with the luminal subtype. However, not all members of the basal-like subtype defined by gene array fulfill the 'triple-negative' immunohistochemistry criteria and vice versa (1). Therefore, we have tried to be consistent in using the term 'triple-negative'.

Recent studies have shown that TNBC has a better pathological complete response (pCR) rate than luminal subtype or non-TNBC but a poorer survival because of higher relapse rates among tumors that are not completely eradicated by chemotherapy $(6,7)$. Other studies have reported that basal keratin and epidermal growth factor receptor (EGFR) are biomarkers of a relatively poorer prognosis among patients with TNBC $(10,11)$. Nevertheless, the prognostic role of EGFR among each subtype after treatment with neoadjuvant chemotherapy remains unclear.

We retrospectively analyzed whether EGFR expression predicts the response to neoadjuvant chemotherapy and is a prognostic marker of survival.

\section{Patients and methods}

One hundred and seventeen Japanese women with stage II or stage III breast cancer who received neoadjuvant chemotherapy between May 1985 and June 2006 were examined. The chemotherapy regimens were as follows: 6 cycles of doxorubicin $\left(50 \mathrm{mg} / \mathrm{m}^{2}\right), 5$-fluorouracil $\left(500 \mathrm{mg} / \mathrm{m}^{2}\right)$, and cyclophosphamide $\left(500 \mathrm{mg} / \mathrm{m}^{2}\right)$ in 24 patients $(20 \%) ; 6$ cycles of the alternate administration of epirubicin $\left(60 \mathrm{mg} / \mathrm{m}^{2}\right), 5$ fluorouracil $\left(500 \mathrm{mg} / \mathrm{m}^{2}\right)$, and cyclophosphamide $\left(500 \mathrm{mg} / \mathrm{m}^{2}\right)$ with docetaxel $\left(75 \mathrm{mg} / \mathrm{m}^{2}\right)$ in 13 patients $(11 \%) ; 6$ cycles of the concurrent administration of doxorubicin $\left(50 \mathrm{mg} / \mathrm{m}^{2}\right)$ and docetaxel $\left(60 \mathrm{mg} / \mathrm{m}^{2}\right)$ in 58 patients $(49 \%)$; and 4 cycles of epirubicin $\left(100 \mathrm{mg} / \mathrm{m}^{2}\right), 5$-fluorouracil $\left(500 \mathrm{mg} / \mathrm{m}^{2}\right)$, and cyclophosphamide $\left(500 \mathrm{mg} / \mathrm{m}^{2}\right)$ followed by 4 cycles of docetaxel $\left(100 \mathrm{mg} / \mathrm{m}^{2}\right)$ in 21 patients $(20 \%)$. Adjuvant hormonal treatment for 5 years was allowed if the disease was 
Table I. Patients characteristics.

\begin{tabular}{|c|c|c|c|c|c|}
\hline & $\begin{array}{c}\text { All } \\
\text { patients }\end{array}$ & $\begin{array}{c}\text { Triple } \\
\text { negative }\end{array}$ & $\mathrm{HER}^{+} / \mathrm{ER}^{-}$ & $\begin{array}{l}\text { Luminal } \\
\qquad\left(\mathrm{ER}^{+}\right)\end{array}$ & P-value \\
\hline $\mathrm{N}(\%)$ & 117 & $28(24 \%)$ & $16(14 \%)$ & $73(62 \%)$ & \\
\hline \multicolumn{6}{|l|}{ Age (year) } \\
\hline Median & 48.4 & 47.9 & 49.8 & 48.4 & 0.8 \\
\hline Range & $27-70$ & $34-68$ & $30-58$ & $34-65$ & \\
\hline \multicolumn{6}{|l|}{ Menopause } \\
\hline Pre & 77 & $16(57 \%)$ & $8(50 \%)$ & $53(73 \%)$ & 0.12 \\
\hline Post & 40 & $12(43 \%)$ & $8(50 \%)$ & $20(27 \%)$ & \\
\hline \multicolumn{6}{|c|}{$\begin{array}{l}\text { Pretreatment } \\
\text { tumor size }(\mathrm{cm})\end{array}$} \\
\hline$\leq 5$ & 33 & $5(18 \%)$ & $3(19 \%)$ & $25(34 \%)$ & 0.17 \\
\hline$>5$ & 84 & $23(82 \%)$ & $13(81 \%)$ & $48(66 \%)$ & \\
\hline \multicolumn{6}{|c|}{$\begin{array}{l}\text { Pretreatment } \\
\text { lymph node status }\end{array}$} \\
\hline Negative & 49 & $6(21 \%)$ & $3(19 \%)$ & $40(55 \%)$ & 0.001 \\
\hline Positive & 68 & $22(79 \%)$ & $13(81 \%)$ & $33(45 \%)$ & \\
\hline
\end{tabular}

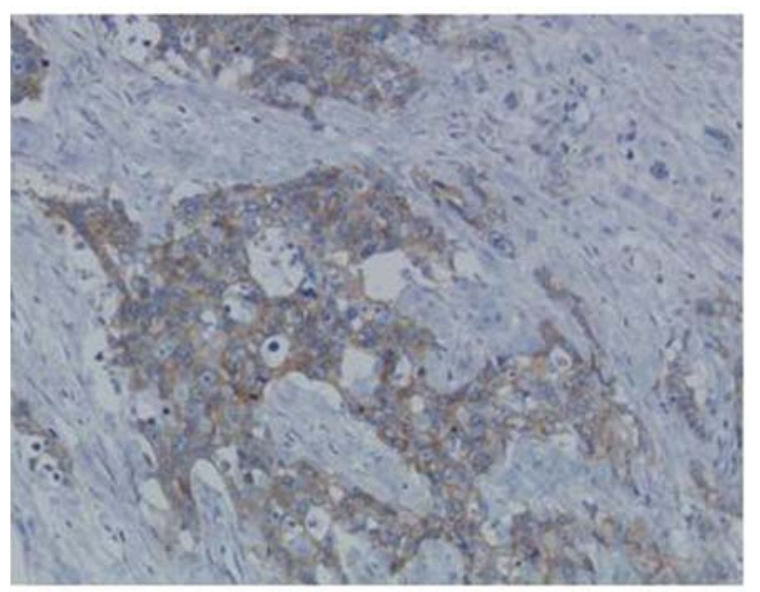

Figure 1. Immunostain for EGFR showing positive membrane and cytoplasmic staining of a tumor cell.

hormone receptor-positive. Systemic and breast examinations were performed before neoadjuvant chemotherapy, before surgery, and every 6 months postoperatively using computed tomography of the chest, ultrasound (US) of the abdomen, magnetic resonance imaging of the brain, and a bone scan, with yearly mammograms and breast US.

Immunohistochemistry methods and definition of breast cancer biological subtypes. Immunohistochemistry (IHC) was performed according to standard procedures on $3-\mu \mathrm{m}$ sections of paraffin-embedded tissue using mouse monoclonal antibody M7047 (Dako, Glostrup, Denmark) for ER and mouse monoclonal antibody M3569 (Dako) for PgR. Nuclear staining $\geq 10 \%$ was considered positive. The expression of HER2 was determined using IHC with a rabbit polyclonal antibody (Dako) on $4-\mu \mathrm{m}$ sections of paraffin-embedded tissue. A staining score of $3^{+}$according to the HercepTest criteria was considered positive. A $2^{+}$ result was only positive if confirmed by fluorescence in situ hybridization with an amplification ratio $\geq 2.0$. The expression of EGFR was determined using IHC with a mouse monoclonal antibody (Novocastra, Wetzlar, Germany) on 3- $\mu \mathrm{m}$ sections of paraffin-embedded tissue. The EGFR stain was considered positive if any cytoplasmic and/or membranous invasive carcinoma cell staining was observed (Fig. 1).

We used immunohistochemical proxies for subtyping and defined TNBC as ER-, PgR-, and HER2-; HER2+/ER- as ER', $\mathrm{PgR}^{-}$and HER2 $2^{+}$; and luminal as $\mathrm{ER}^{+}$and/or $\mathrm{PgR}^{+}$, regardless of the HER2 status.

Statistics. We evaluated the response to chemotherapy according to the Response Evaluation Criteria in Solid Tumor (RECIST) guidelines (12). Overall survival (OS) was measured in months from the date of diagnosis to the date of death or last follow-up. Disease-free survival (DFS) was measured in months from the date of diagnosis to the date of recurrence or last follow-up.

The relations between each subtype and the patients' clinical factors, response rate to adjuvant chemotherapy, and EGFR expression were evaluated using the Fisher's exact test. The Kaplan-Meier product limit method was used to assess the OS and DFS rates. The log-rank test was used to compare survival estimates between the subgroups. Logistic regression analyses were carried out to evaluate the association of EGFR expression with the response to chemotherapy and survival among each subtype. The statistical analyses were performed using StatView ${ }^{\circledR}$ software 
Table II. Breast cancer subtype and response to chemotherapy.

\begin{tabular}{lrccrr}
\hline & Total & Triple-negative & HER2 + ER & Luminal & P-value \\
\hline Number $(\%)$ & $117(100)$ & 28 & 16 & 73 & \\
Clinical response & & & & & \\
$\quad$ Complete response/partial response & $102(87)$ & $25(89)$ & $11(69)$ & $66(90)$ & 0.06 \\
$\quad$ Stable disease/progressive disease & $15(13)$ & $(11)$ & $5(31)$ & $7(10)$ & \\
Pathological response & & & & & 0.6 \\
$\quad$ Complete response & $15(13)$ & $5(18)$ & $2(13)$ & $8(11)$ & \\
$\quad$ Residual disease & $102(87)$ & $23(82)$ & $14(87)$ & $65(89)$ & \\
\hline
\end{tabular}

A

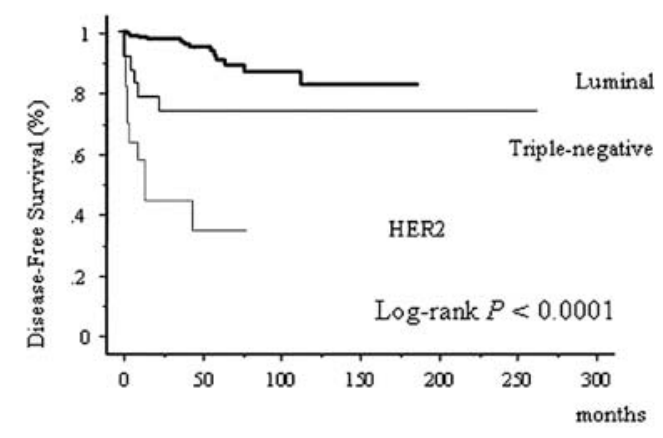

B

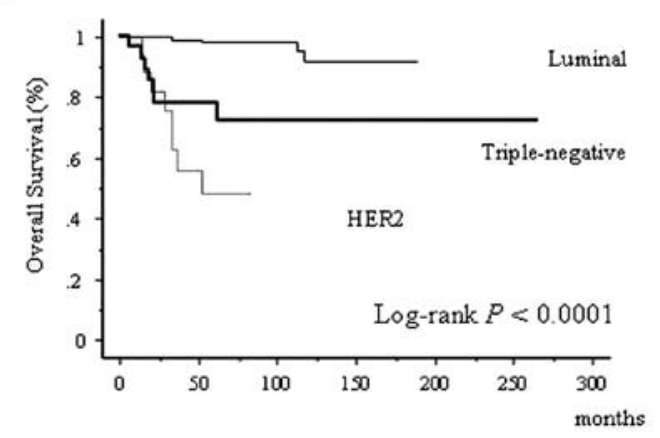

C

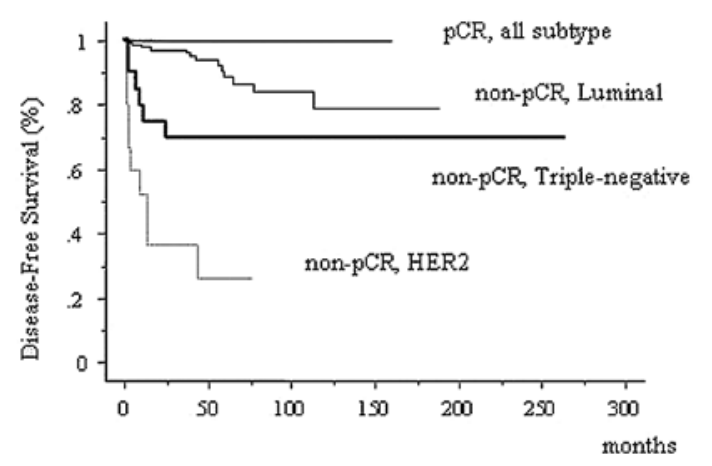

D

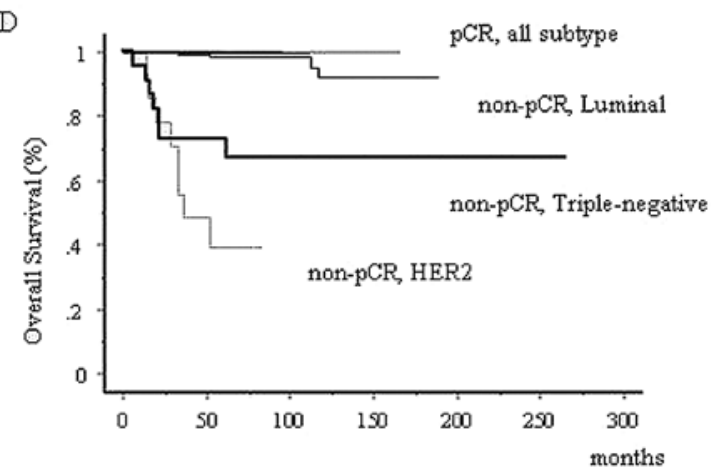

Figure 2. (A) Disease-free survival and (B) overall survival by breast cancer subtype. (C) Disease-free survival and (D) overall survival by pathological complete response vs. residual disease with each subtype.

(version 5, 1998, SAS Institute, Inc., Cary, NC), and $\mathrm{P}<0.05$ was considered statistically significant.

\section{Results}

Patient and tumor characteristics. The median age of the patients was 48 years (range 27-70 years). TNBC accounted for $24 \%$ of the overall breast cancers. Table I lists the patient characteristics and the results of the Fisher's exact tests among each subtype. The positive nodal status before treatment was significantly different between patients with
TNBC or HER2 ${ }^{+} / \mathrm{ER}^{-}$and those with the luminal subtype $(\mathrm{P}=0.001)$, though age, menopausal status and tumor size were similar among the three subtypes (Table I). All the patients received an anthracycline-based regimen, and 93 (79\%) patients also received docetaxel. The regimens did not differ according to subtype.

Response rate to neoadjuvant chemotherapy. One hundred and seven patients $(87 \%)$ had objective clinical responses. Table II lists the clinical response to neoadjuvant chemotherapy and the pCR rates. The response rate did not differ 
Table III. The relationship between EGFR expression and clinical response rates.

Clinical response (complete response + partial response) rates to neoadjuvant chemotherapy

\begin{tabular}{lccrr}
\hline & Total & Triple-negative & HER2 LER $^{-}$ & Luminal \\
\hline EGFR & & & & \\
Positive & $29 / 34(85 \%)$ & $10 / 13(77 \%)$ & $4 / 6(67 \%)$ & $15 / 15(100 \%)$ \\
Negative & $67 / 77(87 \%)$ & $3 / 13(100 \%)$ & $47 / 54(87 \%)$ \\
\hline P-value & 0.81 & 0.03 & 0.89 & 0.06 \\
\hline
\end{tabular}

among the regimens. Twenty-five patients (89\%) with TNBC and 66 patients (90\%) with luminal subtype showed favorable clinical responses to neoadjuvant chemotherapy, compared with eleven patients with HER2/ER - subtype (69\%) $(\mathrm{P}=0.06)$.

Fifteen patients $(13 \%)$ achieved a pCR. The pathological complete response rates did not differ significantly among the subtypes, but the greatest difference was seen between TNBC (18\%) and the luminal subtype (11\%).

Association of subtype with survival. The median follow-up time was 63.4 months. Fig. 2 shows the significant differences in disease-free survival and overall survival among the three subtypes $(\mathrm{P}<0.0001)$. Seven patients $(25 \%)$ with TNBC, 13 patients $(18 \%)$ with luminal subtype and 6 patients $(62 \%)$ with HER2 ${ }^{+} /$ER $^{-}$subtype had distant metastases. Seven patients $(25 \%)$ in the triple-negative group, 5 patients $(7 \%)$ in the luminal group, and 6 patients $(50 \%)$ in the HER2 ${ }^{+} / \mathrm{ER}^{-}$ group died.

None of the patients who achieved a pathological complete response relapsed. The difference between subtypes was particularly apparent after an early follow-up period: all recurrences and deaths among patients with the TNBC or HER2/ER subtypes occurred within 62 months of the date of diagnosis.

EGFR expression and correlation with response to neoadjuvant chemotherapy among the subtypes. One hundred and eleven tumors were available for the evaluation of EGFR expression. Thirty-four (31\%) of the 111 tumors, $13(50 \%)$ of the 26 TNBC tumors, 6 (38\%) of the 16 HER2/ER tumors, and $15(22 \%)$ of the 69 luminal subtype tumors expressed EGFR. The incidence of EGFR expression differed significantly among the groups $(\mathrm{P}=0.02)$.

Table III shows the relationship between EGFR expression and the clinical response rates. Ten $(77 \%)$ of the 13 EGFRpositive TNBC tumors and $13(100 \%)$ of the 13 EGFRnegative TNBC tumors showed an objective clinical response to neoadjuvant chemotherapy. EGFR-positive expression was significantly associated with an unfavorable response $(\mathrm{P}=0.03)$. Fifteen $(100 \%)$ of the 15 EGFR-positive luminal subtype tumors and $47(87 \%)$ of the 54 EGFR-negative luminal subtype tumors showed a clinical response. EGFRpositive expression was associated with a favorable response to chemotherapy among luminal subtype tumors $(\mathrm{P}=0.06)$.

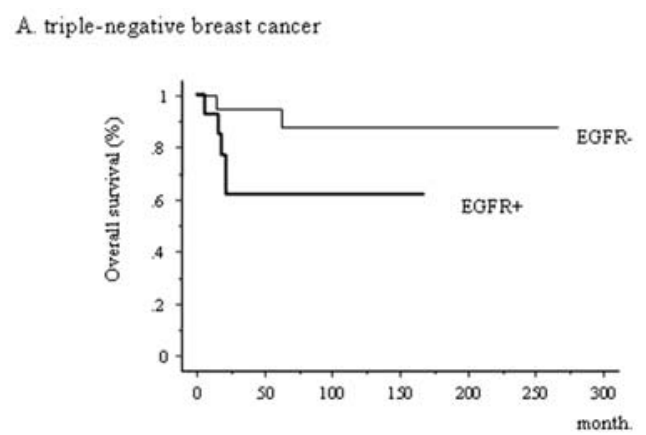

B. HER2+/ER-
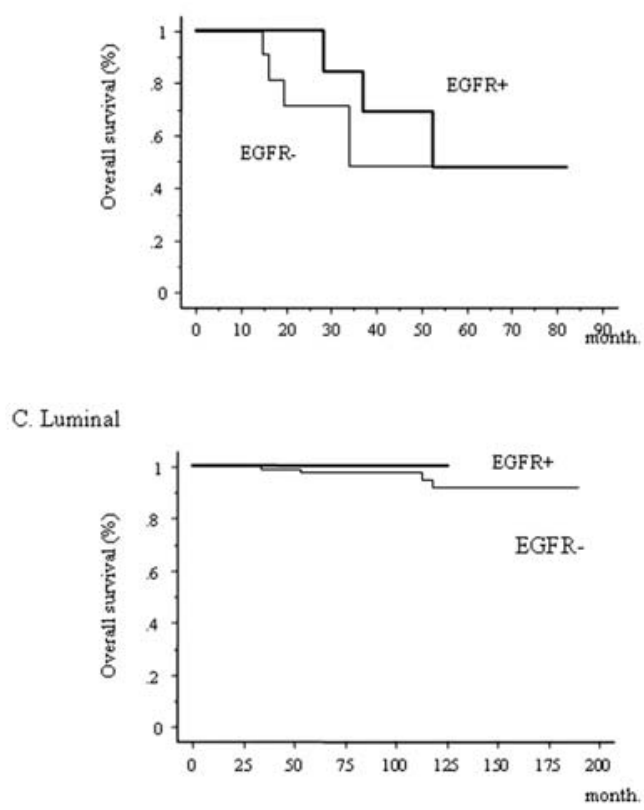

Figure 3. (A) Overall survival by EGFR status with triple-negative breast cancer, (B) HER2 ${ }^{+} / \mathrm{ER}^{-}$subtype and (C) luminal subtype.

Fig. 3 shows the association between EGFR expression and survival in each subtype. Five $(38 \%)$ of the 13 patients with EGFR-positive TNBC tumors and two (15\%) of the 13 patients with EGFR-negative TNBC tumors died. EGFR-positive expression was associated with a poor survival $(\mathrm{P}=0.17)$ among patients with TNBC. The median time-to-death after relapse in patients with EGFR-positive TNBC was markedly shorter (10.8 months; range 7-23 months) than that in patients with EGFR-negative TNBC 
(28.7 months; range 12-62 months). None of the patients with EGFR-positive luminal subtype and 5 of the $54(9 \%)$ patients with EGFR-negative luminal subtype died. EGFRpositive expression was associated with better survival $(\mathrm{P}=0.11)$ among the luminal subtype.

Among the patients with HER2+/ER- tumors, the expression of EGFR was not associated with the response to chemotherapy or survival.

\section{Discussion}

Our retrospective data suggest that the role of EGFR as a predictor of response to chemotherapy and survival was paradoxical. Patients with EGFR-positive TNBC had a significantly less favorable prognosis and a poorer response to neoadjuvant chemotherapy than patients with EGFRnegative TNBC. In contrast, patients with EGFR-positive luminal subtype had a significantly more favorable prognosis and a better response to chemotherapy than patients with EGFR-negative luminal subtype.

EGFR (HER1) is a member of the EGFR family, which includes HER2, HER3 and HER4. This family is important for the proper regulation of many developmental, metabolic, and physiological processes mediated by EGF, transforming growth factor, and many other ligands. In numerous cancers, including breast cancer, glioblastomas, and non-small cell lung cancer, there is often a transforming deregulation of EGFR family kinase activity. This deregulation can be caused by activating mutations, amplification, or the overexpression of EGFR or HER2, although EGFR and HER2 mutations are rare in breast cancer (12). The EGFR pathway is a complex signaling network, and differences in the gene expression levels of its various components can be observed across breast cancer subtypes (13). In our study, the role of EGFR as a predictor of survival and chemosensitivity was contradictory between TNBC and the luminal subtype.

Our data confirmed that patients with TNBC had increased $\mathrm{pCR}$ rates, compared with patients without TNBC, and those who achieved a pCR had an excellent survival; however, patients with residual disease after neoadjuvant chemotherapy had a poorer survival than those with luminal subtype, as previously reported $(6,7,15)$. These findings suggest that effort is needed to improve the poor outcome of patients with TNBC and HER2+/ER- subtypes with residual disease after chemotherapy by continuing appropriate treatment. Trastuzumab is expected to shift the survival curve for the HER2-overexpressing subtype upward, but targeted therapies for TNBC remain unavailable.

EGFR signaling has been inhibited with clinical success, either by using EGFR-directed antibodies (cetuximab) or inhibitors of receptor phosphorylation (gefitinib and erlotinib) $(1,12)$. The activity of gefitinib might be present in subpopulations selected for specific EGFR mutation. In breast cancer patients, the response rate to single EGFR inhibitors has been low; however, these trials were not performed on selected patient populations. Nielsen and Cheang previously reported that the presence of EGFR expression was significantly associated with the expression of basal keratin and a poorer prognosis among patients with TNBC $(10,11)$. The in vitro analyses of Hoadley et al showed that all the basal-like cell lines that were tested were more sensitive to gefitinib and cetuximab, compared with luminal cell lines, and co-treatment with cetuximab and carboplatin was antagonistically synergistic at low-doses of each drug, suggesting a possible good response to EGFR-targeted therapy (14). The activity of cetuximab in the treatment of triple-negative breast cancer is currently being investigated in a metastatic setting (16).

In conclusion, patients with EGFR-positive TNBC had a less favorable response to neoadjuvant chemotherapy and a poorer survival than patients with EGFR-negative TNBC. Especially, all deaths in patients with EGFR-positive TNBC occurred within 22 months of relapse. New thera-peutic options, such as EGFR-targeting or other molecular targeting drugs combined with cytotoxic agents, are urgently needed.

\section{References}

1. Cleator S, Heller W and Coombes RC: Triple-negative breast cancer: therapeutic options. Lancet Oncol 8: 235-244, 2007.

2. Rakha EA, El-Sayed ME, Green AR, Lee AHS, Robertson JF and Ellis IO: Prognostic markers in triple-negative breast cancer. Cancer 109: 25-32, 2007.

3. Bauer KR, Brown M, Cress RD, Parise CA and Caggiano V: Descriptive analysis of estrogen receptor (ER)-negative, progesterone receptor (PR)-negative, and HER2-negative invasive breast cancer, the so-called triple-negative phenotype. Cancer 109: 1721-1728, 2007.

4. Carey LA, Perou CM, Livasy CA, et al: Race, breast cancer subtypes, and survival in the Carolina Breast Cancer Study. JAMA 295: 2492-2502, 2006.

5. Haffty BG, Yang Q, Reiss M, et al: Locoregional relapse and distant metastasis in conservatively managed triple negative early-stage breast cancer. J Clin Oncol 24: 5652-5657, 2006.

6. Carey LA, Dees EC, Sawyer L, et al: The triple negative paradox: primary tumor chemosensitivity of breast cancer subtypes. Clin Cancer Res 13: 2329-2334, 2007.

7. Liedtke C, Mazouni C, Hess KR, et al: Response to neoadjuvant therapy and long-term survival in patients with triple-negative breast cancer. J Clin Oncol 26: 1275-1281, 2008.

8. Sørlie T, Perou CM, Tibshirani R, et al: Gene expression patterns of breast carcinomas distinguish tumor subclasses with clinical implications. Proc Natl Acad Sci USA 98: 10869-10874, 2001.

9. Fan C, Oh DS, Wessels L, et al: Concordance among geneexpression-based predictors for breast cancer. N Engl J Med 355: 560-569, 2006.

10. Nielsen TO, Hsu FD, Jensen K, et al: Immunohistochemical and clinical characterization of the basal-like subtype of invasive breast carcinoma. Clin Cancer Res 10: 5367-5374, 2004.

11. Cheang MC, Voduc D, Bajdik C, et al: Basal-like breast cancer defined by five biomarkers has superior prognostic value than triple-negative phenotype. Clin Cancer Res 14: 1368-1376, 2008.

12. Therasse P, Arbuck SG, Eisenhauer EA, et al: New guidelines to evaluate the response to treatment in solid tumors. European Organization for Research and Treatment of Cancer, National Cancer Institute of the United States, National Cancer Institute of Canada. J Natl Cancer Inst 92: 205-216, 2000.

13. Kumar A, Petri ET, Halmos B, et al: Structure and clinical relevance of the epidermal growth factor receptor in human cancer. Structure and clinical relevance of the epidermal growth factor receptor in human cancer. J Clin Oncol 26: 1742-1751, 2008.

14. Hoadley KA, Weigman VJ, Fan C, et al: EGFR associated expression profiles vary with breast tumor subtype. BMC Genomics 8: 258-276, 2007.

15. Rouzier R, Perou CM, Symmans WF, et al: Breast cancer molecular subtypes respond differently to preoperative chemotherapy. Clin Cancer Res 11: 5678-5685, 2005.

16. Carey LA, Irvin W, Rugo H, et al: TBCRC 001: EGFR inhibition with cetuximab in metastatic triplenegative (basallike) breast cancer. Breast Cancer Res Treat 106 (suppl 1): S32, 2007. 